\title{
THE PRINCIPLE OF EXCHANGE OF STABILITIES FOR COUETTE FLOW
}

\author{
BY \\ ISOM H. HERRON (Department of Mathematical Sciences, Rensselaer Polytechnic Institute, \\ Troy, NY 12180-3590) \\ AND \\ HALIMA N. ALI (Department of Mathematics, Hampton University, Hampton, VA 23668)
}

\begin{abstract}
The eigenvalue problem for the linear stability of Couette flow between two rotating concentric cylinders to axisymmetric disturbances is considered. It is proved that the principle of exchange of stabilities holds when the cylinders rotate in the same direction and the circulation decreases outwards. The proof is based on the notion of a positive operator which is analogous to a positive matrix. Such operators have a spectral property which implies the principle of exchange of stabilities.
\end{abstract}

1. Introduction. The term "exchange of stability" has a long history dating back to the late 1800's, but the now conventional understanding of the principle of exchange of stabilities (PES) (i.e., all non-decaying disturbances are non-oscillatory in time) seems to have been introduced by Jeffreys [10]. A much investigated motion is Couette flow between rotating cylinders [24] and careful experiments on the onset of instability by Taylor [25], [26] and others [2], [19] have confirmed that the instability of this flow sets in as a stationary secondary flow. The purpose here is to prove PES in the following sense:

Principle of Exchange of Stabilities (PES): The first unstable eigenvalue has imaginary part equal to zero.

This is now taken as the "weak" form of PES [28], [17]. A "strong" form of PES in which all the eigenvalues are real has been proven for some other problems [1], [3], [7]. Thus, Yih [29] asserted, and seemingly proved, the strong form of PES for Couette flow between two cylinders. To our knowledge, no error has been detected in his proof. However, DiPrima \& Hall [5] have produced calculations of complex higher eigenvalues which would contradict the strong form of PES. They also provided asymptotic/perturbation arguments to buttress their calculations. The results of DiPrima \& Hall are consistent with the weak form of PES.

Received March 1, 2001.

2000 Mathematics Subject Classification. Primary 76E07; Secondary 47B25, 47N20.

Key words and phrases. Hydrodynamic stability, Couette flow.

E-mail address: herroi@rpi.edu

E-mail address: halima.ali@hamptonu.edu 
The proof to be presented here is based on two methods. One, due to Weinberger [28] was applied without complete success to the present problem. As he applied it then, the implied calculations were so complicated as to defy interpretation. Another method, developed by Rabinowitz, was applied successfully to the Bénard problem [21]. By combining the two methods, using the different techniques and more refined estimates introduced here, the proof goes through. However, by using asymptotic expansions, Weinberger did prove the PES for the narrow-gap case. His technique is also noteworthy because it can be generalized to boundary layer flow. In fact, Herron [9] used it to give the first mathematical justification of PES for Görtler flow with a free surface.

The techniques employed are based on the notion of a positive operator, that is one which maps a cone in a Banach space into itself. In this approach, the Banach space is a Hilbert space $H_{0}$, and the cone is the set of nonnegative functions in $H_{0}$. The reader is referred to the earlier articles, [28], [21] (and also [13], [18]) for the foundations but to apply the methods, it is shown that the governing equations may be adapted to the form treated earlier [28], [21].

In the next section the basic formulation is given. In the succeeding section, the earlier approach is presented. In the concluding sections the governing disturbance equations are written in operator form and the desired result is proved.

2. Basic formulation. Consider the steady motion of a viscous incompressible fluid between two coaxial cylinders with radii $R_{1}, R_{2}\left(R_{2}>R_{1}\right)$, rotating about their common axis with respective angular velocities $\Omega_{1}, \Omega_{2}$. Let $(r, \theta, z)$ be cylindrical polar coordinates with $z$-axis along the axis of the cylinders. A flow is possible of the form

$$
u_{r}=u_{z}=0, \quad u_{\theta}=V(r), \quad p=P(r),
$$

with

$$
V=A_{1} r+A_{2} / r
$$

so that $A_{1}$ and $A_{2}$ are given by

$$
A_{1}=-\Omega_{1} \eta^{2} \frac{1-\mu / \eta^{2}}{1-\eta^{2}} \text { and } A_{2}=\Omega_{1} R_{1}^{2} \frac{1-\mu}{1-\eta^{2}},
$$

where

$$
\mu=\Omega_{2} / \Omega_{1} \text { and } \eta=R_{1} / R_{2} \text {. }
$$

The constants $\mu$ and $\eta$ may be called the kinematical ratio and the geometrical ratio of the steady motion respectively. The motion conforming to (2.2) and (2.3) is the Couette flow.

The first experiments on the onset of instability in the viscous Couette flow between rotating cylinders are those of Taylor [25], [26]. His experiments showed that this instability leads to a new steady secondary axisymmetric flow in the form of regularly spaced vortices in the axial direction, commonly known as Taylor vortices. In the narrow-gap case, Taylor found an explicit analytic expression for the stability critcrion. Synge [23] gave a relatively simpler proof to Taylor's analytic expression without the assumption on the gap. He showed that the real part of the eigenvalue $\sigma$ occurring in an "exponential time factor", i.e. a normal mode analysis, can be expressed as the ratio of certain 
positive definite integrals, in which the integrands contain the product of an eigenfunction or one of its derivatives by the complex conjugate. He went on to show that if $\Omega_{2} R_{2}^{2}>\Omega_{1} R_{1}^{2}>0$ (i.e. $\mu>\eta^{2}, \Omega_{1}>0$ ), the signs of these integrals are known without the knowledge of the eigenfunctions and he found that the steady motion is stable to disturbances that are independent of the azimuthal angle $\theta$ and periodic in the direction of the generators of the cylinders under the above condition. However, his proof does not lead to any conclusions about the behavior of the imaginary part of $\sigma$. The Synge result has been extended to finite amplitude disturbances by Joseph \& Hung [12]. As for instability results, rigorous proof was first provided by Velte [27], and by Yudovich [30], [31]. An important nonlinear extension to the bifurcated state has recently been enunciated by Schneider [22].

On the basis of experimental evidence, Taylor assumed that the PES is valid for the problem he considered. This assumption has been made by many subsequent investigators of the subject (at least when the cylinders rotate in the same direction) but a theoretical proof had never been advanced until Yih. Yih's proof is rather complicated and is also controversial as indicated by the work of DiPrima \& Hall. It is the objective of the present work to give a more direct proof that the PES holds for Couette flow in the case $0<\mu<\eta^{2}$. The rest of this section is devoted to the presentation of the basic disturbance equations.

Consider an infinitesimal perturbation of the basic flow given by equations (2.1) and describe the perturbed flow by

$$
\mathbf{u}=\left(u^{\prime}, V+v^{\prime}, w^{\prime}\right) \text { and } p=P+p^{\prime} .
$$

The linearized equations of motion then follow from the Navier-Stokes equations and the continuity equation. Thus, for disturbances that are periodic in the axial direction, one can look for separated solutions of the form

$$
u^{\prime}=u(r) e^{\sigma t+i \alpha z}
$$

with similar expressions for the other components of the velocity and the pressure, where $\alpha$ is the wave number of the disturbance in the axial direction and $\sigma$ is the growth rate. Substituting in the linearized disturbance equations and using the axial momentum equation and the continuity equation to eliminate $p$ and $w$, after some rearranging, one obtains the equations in dimensionless form (cf. [1])

$$
\begin{aligned}
\left(D D_{*}-\alpha^{2}-\sigma\right)\left(D D_{*}-\alpha^{2}\right) u & =-\alpha^{2} T\left(\frac{1}{r^{2}}-\kappa\right) v \\
\left(D D_{*}-\alpha^{2}-\sigma\right) v & =u
\end{aligned}
$$

where $D_{*}=\frac{d}{d r}+\frac{1}{r}$ and $D=\frac{d}{d r}$,

$$
T=-\frac{4 A_{1} A_{2}}{\nu^{2}} R_{2}^{2} \quad \text { and } \quad \kappa=-\frac{A_{1} R_{2}^{2}}{A_{2}} .
$$

Solutions of equations (2.4)-(2.5) must be sought which satisfy the boundary conditions appropriate for no slip on the cylindrical walls $r=\eta$ and $r=1$. These conditions are 
that all three components of the velocity vanish on the wall; thus,

$$
u=v=D u=0 \text { for } r=\eta \text { and } r=1 \text {. }
$$

The parameter $T$ that appears in (2.4) is the Taylor number. In terms of $\mu$ and $\eta$, it has the form

$$
T=-\frac{4 A_{1} A_{2}}{\nu^{2}} R_{2}^{2}=\frac{4 R^{2}(1-\mu)\left(1-\mu / \eta^{2}\right)}{\left(1-\eta^{2}\right)^{2}}
$$

where $R$ is the Reynolds number based on the inner cylinder. Notice first that $T$ is only positive if $\mu<\eta^{2}$. It is precisely the condition $\mu<\eta^{2}$ that is necessary and sufficient for instability in an inviscid fluid and is necessary for instability in a viscous fluid. Second, the Taylor number has the form of the square of Reynolds number times a function of $\mu$ and $\eta$. Notice also that the parameter $k$ in equation $(2.4)$ may be written as

$$
r=-\frac{A_{1} R_{2}^{2}}{A_{2}}=\frac{1-\mu / \eta^{2}}{1-\mu} .
$$

Thus, when the cylinders rotate in the same direction, $0<k<1$.

3. The problem of PES and the method of Positive Operators. The idea of the method of solution is based on the notion of a positive operator [13], [8]. a generalization of a positive matrix. that is, one with all of its entries positive. Such matrices have the property that they possess a single greatest positive eigenvalue, identical to the spectral radius. To apply the method the resolvent of the linearized stability operator is analyzed. This resolvent is in the form of compositions of certain integral operators. When the Green's function kernels for these operators are all nomnegative, the resulting operator is termed positive. Explicitly, we have:

Definition 1. Let $\mathfrak{B}$ be a Banach space and let there be closed set $\mathfrak{K}$ with the properties:

(1) If $v_{1}, v_{2}$ belong to $\mathfrak{K}$ and $c_{1}, c_{2}$ are real non-negative scalars, then $c_{1} v_{1}+c_{2} v_{2} \in \mathfrak{K}$.

(2) If $v_{1} \in \mathfrak{K}$ and $-v_{1} \in \mathfrak{K}$, then $v_{1}=0$.

Then we call $\mathfrak{K}$ a cone.

Definition 2. [13] A positive operator is a bounded linear mapping taking $\mathfrak{K}$ into itself.

A basic theorem for this work is the

Krein-Rutman Theorem. [18] Let $A$ be a compact linear operator in a Banach space $\mathfrak{B}$. Assume $A$ maps a cone $\mathfrak{K}$ into itself and that $A$ has a nonzero element in its spectrum. Then $A$ has a positive eigenvalue $\lambda$ with $\lambda \geq|\mu|$ for any other $\mu$ in the spectrum of $A$. Moreover, there is at least one cigenvector $\phi$ of $A$ in $\mathfrak{K}$ and at least one eigenvector $\psi$ of $A^{*}$ in $\mathfrak{K}^{*}$.

The formulation proceeds along the following lines [28]. Consider equations (2.4) and (2.5). Let the inverses of the differential operators be defined as

$$
\begin{gathered}
\Gamma(\sigma)=-\left[D D_{*}-\alpha^{2}-\sigma\right]^{-1} \\
G(\sigma)=\left[\left(D D_{*}-\alpha^{2}\right)\left(D D_{*}-\alpha^{2}-\sigma\right)\right]^{-1} .
\end{gathered}
$$


where the domains of the differential operators are those given by the boundary conditions (2.6). (In the next section these operators will be considered abstractly. Certain restrictions and extensions of the operators will also be introduced.) Consequently it is possible to reformulate (2.4)-(2.5) as

$$
u-K(\sigma) u=0
$$

where

$$
K(\sigma)=\alpha^{2} T G(\sigma)\left[\left(\frac{1}{r^{2}}-\kappa\right) \Gamma(\sigma)\right] .
$$

Suppose $K(\sigma)$ depends analytically on $\sigma$ in a certain right half of the complex plane. When the cylinders rotate in the same direction, and the circulation decreases outwards, $\frac{1}{r^{2}}-\kappa>0$.

By this approach, examine the resolvent

$$
[I-K(\sigma)]^{-1}=\left\{I-\left[I-K\left(\sigma_{0}\right)\right]^{-1}\left[K(\sigma)-K\left(\sigma_{0}\right)\right]\right\}^{-1}\left[I-K\left(\sigma_{0}\right)\right]^{-1},
$$

so that if for all real $\sigma_{0}$ greater than some $a$,

(P1): $\left[I-K\left(\sigma_{0}\right)\right]^{-1}$ is positive, and

(P2): $K(\sigma)$ has a power series expansion about $\sigma_{0}$ with positive coefficients, that is, $\left(-\frac{d}{d \sigma}\right)^{n} K\left(\sigma_{0}\right)$ is positive for all $n$; then the right hand side of $(3.5)$ has an expansion in $\left(\sigma_{0}-\sigma\right)$ with positive coefficients.

With the theory previously developed, it follows that there exists a real eigenvalue $\sigma_{1} \leq a$ such that the spectrum of $K(\sigma)$ lies in the set $\left\{\sigma \in \mathbf{C} \mid \operatorname{Re}(\sigma) \leq \sigma_{1}\right\}$. This result is equivalent to the weak form of the principle of exchange of stabilities. This is true by virtue of the Krein-Rutman Theorem about positive operators.

Next, go on to show that $\Gamma(\sigma)$ is an integral operator whose kernel $\Gamma(r, \rho ; \sigma)$ is the Laplace transform of the Green's function $\Gamma^{*}(r, \rho ; t)$ for the initial-boundary problem

$$
\begin{gathered}
\left(-\frac{\partial^{2}}{\partial r^{2}}-\frac{1}{r} \frac{\partial}{\partial r}+\frac{1}{r^{2}}+\alpha^{2}+\frac{\partial}{\partial t}\right) u=f, \\
u(\eta, t)=u(1, t)=u(r, 0)=0 .
\end{gathered}
$$

Since there is a maximum principle associated with (3.6)-(3.7), $f \geq 0$, implies $u \geq 0$. (It also follows by directly analyzing the Laplace transform [6].) Thus, $\Gamma^{*}(r, \rho, t) \geq 0$. Since

$$
\Gamma(r, \rho ; \sigma)=\int_{0}^{\infty} e^{-\sigma t} \Gamma^{*}(r, \rho ; t) d t
$$

it follows that

$$
\left(-\frac{d}{d \sigma}\right)^{n} \Gamma(r, \rho ; \sigma)=\int_{0}^{\infty} t^{n} e^{-\sigma t} \Gamma^{*}(r, \rho ; t) d t \geq 0
$$

for all $n$ and for all $\sigma>-\alpha^{2}$. 
However, the verification of hypothesis (P2) is incomplete [28]. The problem is the integral operator $G(\sigma)$, which is not as easily analyzed as $\Gamma(\sigma)$. The kernel of the integral operator is $G(r, \rho ; \sigma)$, the Laplace transform of the Green's function of the time dependent problem

$$
\begin{gathered}
\left(-\frac{\partial^{2}}{\partial r^{2}}-\frac{1}{r} \frac{\partial}{\partial r}+\frac{1}{r^{2}}+\alpha^{2}+\frac{\partial}{\partial t}\right)\left(-\frac{\partial^{2}}{\partial r^{2}}-\frac{1}{r} \frac{\partial}{\partial r}+\frac{1}{r^{2}}+\alpha^{2}\right) u=f \\
u=\frac{\partial u}{\partial r}=0 \text { at } r=\eta \text { and } r=1 \\
u=0 \text { at } t=0 .
\end{gathered}
$$

As in the above argument, the inequalities $\left(-\frac{d}{d \sigma}\right)^{n} G(\sigma)>0$ would follow if it could be shown that the Green's function $G^{*}(r, \rho ; t)$ of the problem $(3.10)-(3.12)$ is nonnegative. Once this is done, it would follow from the product rule for differentiation that the operator

$$
K(\sigma)=\alpha^{2} T G(\sigma)\left[\left(\frac{1}{r^{2}}-\kappa\right) \Gamma(\sigma)\right]
$$

has the property (P2). The Green's function $G(r, \rho ; \sigma)$ can be written explicitly in terms of Bessel functions, but the expression is, seemingly, too complicated to verify (P2) directly.

Thus, the verification of conditions (P1) and (P2) is incomplete [28]. One reason why is that the integral operator $G(\sigma)$ is the inverse of a fourth order differential operator with variable coefficients. The major difficulty, however, lies in the boundary conditions. which do not allow the differential operator (and consequently its inverse) to be factored naturally in the abstract sense. This difficulty is dealt with in the next section by means of the generalized inverse. It is proved that the necessary factorization may bo accomplished by introducing a projection, which connects $\Gamma(\sigma)$ and $G(\sigma)$ in a significant way. The use of totally positive kernels also plays a role in the analysis. Some information of this type was known before the results of [28]. The condition (P1) was proved in the papers [30], [31] using totally positive kernels. It was proved there that $K(\sigma)$ for all real positive $\sigma$ (and even for negative $\sigma$ sufficiently small in absolute value) is a totally positive operator. This was the means of establishing rigorously that instability occurs above a certain Taylor number.

4. Abstract formulation. In operator form the differential equations (2.4)-(2.5) may be written as

$$
\begin{gathered}
\left(M^{*} M+\sigma M\right) u+\alpha^{2} T\left(\frac{1}{r^{2}}-\kappa\right) v=0 . \\
u+(\tilde{M}+\sigma) v=0,
\end{gathered}
$$


where

$$
\begin{array}{ll}
M u=\left(-D D_{*}+\alpha^{2}\right) u:=m u, & u \in \operatorname{dom} M \\
M^{*} M u=m^{2} u, & u \in \operatorname{dom}\left(M^{*} M\right) \\
\tilde{M} v=m v, & v \in \operatorname{dom} \tilde{M} .
\end{array}
$$

The domains are contained in $H_{0}$, where

$$
H_{0}=\left\{\left.\varphi\left|\int_{\eta}^{1} r\right| \varphi\right|^{2} d r<\infty\right\}
$$

with inner product

$$
\langle\varphi, \psi\rangle_{0}=\int_{\eta}^{1} r \varphi(r) \bar{\psi}(r) d r, \varphi, \psi \in H_{0}
$$

and norm

$$
\|\varphi\|_{0}=\left(\langle\varphi, \varphi\rangle_{0}\right)^{1 / 2}
$$

The domains are given as follows:

$$
\begin{aligned}
\operatorname{dom} M^{*} & =\left\{\varphi \in H_{0} \mid m \varphi \in H_{0}\right\}, \\
\operatorname{dom} M & =\left\{\varphi \in \operatorname{dom} M^{*} \mid \varphi(\eta)=\varphi(1)=D \varphi(\eta)=D \varphi(1)=0\right\}, \\
\operatorname{dom} \tilde{M} & =\left\{\varphi \in \operatorname{dom} M^{*} \mid \varphi(\eta)=\varphi(1)=0\right\} .
\end{aligned}
$$

Thus, with the above definitions understood, it is not difficult to verify the following properties of the operators just defined (cf. [16]).

REMARK 1. $M$ is closed, symmetric, but not maximal and hence not invertible. Moreover, $M$ is positive definite, that is

$$
\langle M \varphi, \varphi\rangle_{0} \geq \alpha^{2}\|\varphi\|_{0}^{2}, \quad \varphi \in \operatorname{dom} M, \quad \alpha \neq 0 .
$$

The case $\alpha=0$ is excluded because stability is known to hold in this case.

REMARK 2. $M^{*}$ is the adjoint of $M$ and has no boundary conditions. The twodimensional null space of $M^{*}, \operatorname{ker} M^{*}$, has the basis

$$
\mathbf{q}(r)=\left[\begin{array}{c}
I_{1}(\alpha r) \\
K_{1}(\alpha r)
\end{array}\right],
$$

where $I_{1}$ and $K_{1}$ are the modified Bessel functions of order one of the first and the second kind respectively.

REMARK 3. $\tilde{M}$ is a maximal selfadjoint, positive definite extension of $M$. Furthermore, $\Gamma(\sigma)=(\tilde{M}+\sigma)^{-1}$ exists for

$$
\sigma \notin \Sigma_{\alpha}=\left\{\sigma \in \mathbf{C} \mid \operatorname{Re}(\sigma) \leq-\alpha^{2}, \operatorname{Im}(\sigma)=0\right\},
$$

and $\|\Gamma(\sigma)\|_{0}^{-1}>\left|\sigma+\alpha^{2}\right|$, for $\operatorname{Re}(\sigma)>-\alpha^{2}[16$, p. 272]. 
A formulation similar to (4.1)-(4.2) was made by DiPrima \& Habetler [4]. They were able to prove a completeness theorem for the cigenfunctions in the following sense. Let $\mathfrak{H}$ be the Hilbert space $\mathfrak{H}=H_{0} \times H_{0}$ (where $H_{0}$ is the space previously defined), and $\Phi=\left(\begin{array}{c}\varphi_{1} \\ \varphi_{2}\end{array}\right) \in \mathfrak{H}$, with inner product

$$
(\Phi, \Psi)=\left\langle\varphi_{1}, \psi_{1}\right\rangle_{0}+\left\langle\varphi_{2}, \psi_{2}\right\rangle_{0} . \Phi \in \mathfrak{H} . \Psi \in \mathfrak{H} .
$$

Write $\mathrm{L} \Phi=\left(\mathrm{L}_{s}+\mathrm{B}\right) \Phi=\lambda \mathrm{M} \Phi$, where

$$
\mathrm{L}_{s}=\left(\begin{array}{cc}
M^{*} M & 0 \\
0 & \tilde{M}
\end{array}\right), \quad \mathrm{B}=\left(\begin{array}{cc}
0 & \alpha^{2} T\left(\frac{1}{r^{2}}-\kappa\right) \\
1 & 0
\end{array}\right), \quad \mathrm{I}=\left(\begin{array}{cc}
\tilde{M} & 0 \\
0 & 1
\end{array}\right)
$$

Then completeness holds in the space $\mathfrak{H}_{M}$ which is imbedded in $\mathfrak{H}$, by completing the pre-Hilbert space ( dom $\mathrm{M},[]$,$) , where [\Phi, \Psi]=(\Phi, M \Psi)=\left\langle\varphi_{1}, \tilde{N} \psi_{1}\right\rangle_{0}+\left\langle\varphi_{2}, \psi_{2}\right\rangle_{0}$ for $\Phi \in \operatorname{dom} M, \Psi \in \operatorname{dom} M$. Their analysis was performed by introducing the self-adjoint extension of $\mathrm{M}^{-1} \mathrm{~L}_{s}$ in $\mathfrak{H}_{\mathrm{M}}$.

However, going beyond [4], a factorization of $\left(M^{*} M\right)^{-1}$ is needed in the current work. Owing to the fact that $M$ is not invertible, we will employ a generalized inverse [20] to $M$ called $M I^{\dagger}$. First, the projection operator $Q$ onto the $\operatorname{ker} M^{*}$ is defined by

$$
(Q \psi)(r)=\int_{\eta}^{1} \rho g_{Q}(r, \rho) \psi(\rho) d \rho, \quad \psi \in H_{0},
$$

where

$$
g_{Q}(r, \rho)=\mathbf{q}^{T}(r)\left[\int_{\eta}^{1} s \mathbf{q}(s) \mathbf{q}^{T}(s) d s\right]^{-1} \mathbf{q}(\rho) .
$$

Then $g^{\dagger}$, the generalized Green's function, satisfies

$$
\begin{gathered}
\left(-\frac{\partial^{2}}{\partial r^{2}}-\frac{1}{r} \frac{\partial}{\partial r}+\frac{1}{r^{2}}+\alpha^{2}\right) g^{\dagger}(r, \rho)=\delta(r-\rho)-g_{Q}(r, \rho), \\
g^{\dagger}(\eta, \rho)=\frac{\partial}{\partial r} g^{\dagger}(\eta, \rho)=g^{\dagger}(1, \rho)=\frac{\partial}{\partial r} g^{\dagger}(1, \rho)=0
\end{gathered}
$$

so that

$$
\left(M^{\dagger} \varphi\right)(r)=\int_{\eta}^{1} \rho g^{\dagger}(r, \rho) \varphi(\rho) d \rho .
$$

Some properties of $M^{\dagger}$ are

$$
\begin{gathered}
M M^{\dagger}=I-Q, \\
M^{\dagger} M=I .
\end{gathered}
$$

since $\operatorname{ker} M$ is trivial.

Lemma 1. $M I^{\dagger}=\tilde{M}^{-1}(I-Q)$. 
Proof. Since $\tilde{M}$ is an invertible extension of $M, \tilde{M}^{-1} M=I$ on dom $M$, therefore operating on (4.9) with $\tilde{M}^{-1}$

$$
\tilde{M}^{-1} M M^{\dagger}=\tilde{M}^{-1}(I-Q)
$$

and

$$
\tilde{M}^{-1} M M^{\dagger}=I M I^{\dagger}=M I^{\dagger}
$$

so

$$
M^{\dagger}=\tilde{M}^{-1}(I-Q) .
$$

LEMma 2. The only solution of (4.1)-(4.2) is the trivial solution, when $T=0$, and $\sigma \notin \Sigma_{\alpha}$.

Proof. Suppose $T=0$, then (4.1)-(4.2) reduces to

$$
\begin{gathered}
\left(M^{*} M+\sigma M\right) u=0 . \\
u+(\tilde{\Lambda}+\sigma) v=0 .
\end{gathered}
$$

Taking inner products of $(4.12)$ with $u$ we see

$$
\left\langle M^{*} M u, u\right\rangle_{0}=-\sigma\langle M u, u\rangle_{0},
$$

or

$$
\begin{aligned}
-\sigma\langle M u, u\rangle_{0} & =\left\langle M^{*} M u, u\right\rangle_{0}=\langle M u, M u\rangle_{0}=\|M u\|_{0}^{2} \geq\langle M u, u\rangle_{0}^{2} /\|u\|_{0}^{2} \\
& \geq \alpha^{2}\langle M u, u\rangle_{0},
\end{aligned}
$$

by Remarks 1 and 2. Thus, $\sigma$ is real and $\sigma \leq-\alpha^{2}$. Hence when $\sigma \notin \Sigma_{\alpha}$, $\Rightarrow u=0 \Rightarrow$ $v=0$, by (4.13) and Remark 3 .

Lemma 3. The operator $L=M^{*} M+\sigma M$ has an inverse, positive for all real $\sigma_{0}>-\alpha^{2}$. The inverse has a power series expansion about $\sigma_{0}$ in powers of $\sigma_{0}-\sigma$ with positive coefficients.

Proof. On the basis of Lemma 2, conclude that $L$ is invertible for $\sigma \notin \Sigma_{\alpha}$. Define

$$
L^{-1}=G(\sigma)=\left(M^{*} M+\sigma M\right)^{-1}=\left(M^{*} M\right)^{-1}(I+\sigma B)^{-1},
$$

where $B=M\left(M^{*} M\right)^{-1}$ is a bounded operator. Note that the meaning of $B$ is different than used in (4.3).

It has been shown [28], $((3.8),(3.9))$ that $\Gamma(\sigma)=(\tilde{M}+\sigma)^{-1}$ is a positive operator for all real $\sigma_{0}>-\alpha^{2}$, and that $\Gamma(\sigma)$ has a power series expansion about $\sigma_{0}$ in $\left(\sigma_{0}-\sigma\right)$ with positive coefficients, i.e. $\left(-\frac{d}{d \sigma}\right)^{n} \Gamma\left(\sigma_{0}\right)$ is positive for all $n$. Thus the expansion

$$
\begin{gathered}
\Gamma(\sigma)=\Gamma\left(\sigma_{0}\right)\left[I-\left(\sigma_{0}-\sigma\right) \Gamma\left(\sigma_{0}\right)\right]^{-1} \\
=\Gamma\left(\sigma_{0}\right)\left[I+\left(\sigma_{0}-\sigma\right) \Gamma\left(\sigma_{0}\right)+\left(\sigma_{0}-\sigma\right)^{2}\left(\Gamma\left(\sigma_{0}\right)\right)^{2}+\ldots\right]
\end{gathered}
$$

is valid for $\left|\sigma_{0}-\sigma\right|\left\|\Gamma\left(\sigma_{0}\right)\right\|_{0}<1$. The coefficients are positive operators when $\sigma_{0}>-\alpha^{2}$. Analogously, by (4.14),

$$
\left(M^{*} M+\sigma M\right)^{-1}=\left(M^{*} M I+\sigma_{0} M I\right)^{-1}\left[I-\left(\sigma_{0}-\sigma\right) M\left(M^{*} M I+\sigma_{0} M I\right)^{-1}\right]^{-1}
$$


so that

$$
\begin{gathered}
G(\sigma)=G\left(\sigma_{0}\right)\left[I-\left(\sigma_{0}-\sigma\right) M I G\left(\sigma_{0}\right)\right]^{-1}= \\
G\left(\sigma_{0}\right)\left[I+\left(\sigma_{0}-\sigma\right) M I G\left(\sigma_{0}\right)+\left(\sigma_{0}-\sigma\right)^{2}\left(M G\left(\sigma_{0}\right)\right)^{2}+\ldots\right] .
\end{gathered}
$$

for $\left|\sigma_{0}-\sigma\right|\left\|\Lambda I G\left(\sigma_{0}\right)\right\|_{0}<1, \sigma \notin \Sigma_{\alpha}, \sigma_{0}>-\alpha^{2}$.

It is still necessary to establish the positivity of the coefficients in the expansion for $G(\sigma)$. Observe, also from (4.14), that

$$
G\left(\sigma_{0}\right)=\left(M^{*} M\right)^{-1}\left[I+\left(-\sigma_{0}\right) B+\left(-\sigma_{0}\right)^{2} B^{2}+\ldots\right]
$$

is defined for $\left|\sigma_{0}\right|\|B\|_{0}<1$. If it can be shown that the coefficients in the above expansion (4.16) of $G\left(\sigma_{0}\right)$ in powers of $\left(-\sigma_{0}\right)$ are positive, then it follows that $G\left(\sigma_{0}\right)$ is a positive operator for real $\sigma_{0}$ in the interval $-\alpha^{2}<\sigma_{0}<\|B\|_{0}^{-1}$. Then by a process of analytic continuation, which will be described, the positivity of $G\left(\sigma_{0}\right)$ for all real $\sigma_{0}>-\alpha^{2}$ will be proved.

The process thus begins to establish that $G(\sigma)$ in $(4.15)$ has positive expansion coefficients. This is done by re-examining the expansion of $G\left(\sigma_{0}\right)$ (4.16). We have, from (4.11), that

$$
M^{\dagger *}=\left[\tilde{M}^{-1}(I-Q)\right]^{*}=(I-Q) \tilde{M}^{-1}
$$

since both $\tilde{M}^{-1}$ and $Q$ are selfadjoint. Thus we have the equivalent factorizations

$$
M^{\dagger} M I^{\dagger *}=\tilde{M}^{-1}(I-Q)(I-Q) \tilde{M}^{-1}=M^{\dagger} \tilde{M}^{-1}=\tilde{I}^{-1} M I^{\dagger *}=\tilde{M}^{-1}(I-Q) \tilde{I}^{-1},
$$

since $I-Q$ is a projection. In fact, since $Q$ is the projection onto $\operatorname{ker} M^{*}, I-Q$ is the projection onto ran $M$. the range of $M$. But, by definition of the generalized inverse,

$$
M^{\dagger} M I^{\dagger *}=\left(M^{*} M I\right)^{\dagger}=\left(M I^{*} M\right)^{-1},
$$

since $M^{*} M$ is invertible, being positive (definite) selfadjoint. Thus

$$
B=M\left(M^{*} M\right)^{-1}=M^{\dagger *},
$$

and

$$
\|B\|_{0}=\left\|(I-Q) \tilde{I}^{-1}\right\|_{0} \leq\left\|\tilde{\Lambda}^{-1}\right\|_{0},
$$

since $I-Q$ is a projection.

Then the expansion of $G\left(\sigma_{0}\right)$ (4.16), may be re-written, using (4.19), as

$$
\begin{gathered}
G\left(\sigma_{0}\right)=\left(M^{*} M\right)^{-1}+\left(-\sigma_{0}\right)\left(M^{*} M\right)^{-1} M\left(M^{*} M\right)^{-1}+ \\
+\left(-\sigma_{0}\right)^{2}\left(M^{*} M\right)^{-1}\left(M\left(I^{*} M\right)^{-1}\right)^{2}+\ldots \\
=\left(M^{*} M\right)^{-1}+\left(-\sigma_{0}\right) M I^{\dagger} \tilde{I}^{-1} M^{\dagger *}+\sigma_{0}^{2}\left(M^{\dagger} M I^{\dagger *}\right)\left(M I^{\dagger *}\right)^{2}+\ldots
\end{gathered}
$$

By (4.11) and (4.17) it follows that

$$
\begin{aligned}
G\left(\sigma_{0}\right)= & \left(M^{*} M\right)^{-1}+\left(-\sigma_{0}\right) \tilde{M}^{-1}(I-Q) \tilde{M}^{-1}(I-Q) \tilde{M}^{-1} \\
& +\sigma_{0}^{2} \tilde{M}^{-1}(I-Q) \tilde{M}^{-1}\left((I-Q) \tilde{M}^{-1}\right)^{2}+\ldots
\end{aligned}
$$


It is a well known result of the stability literature [11, Appendix D], [21, p. 370], that $\tilde{M}^{-1}$ has a non-negative Green's function kernel. Similar to being identified as $\Gamma(0)$, this follows from the fact that the operator

$$
-M^{*}=\frac{d^{2}}{d r^{2}}+\frac{1}{r} \frac{d}{d r}-\left(\frac{1}{r^{2}}+\alpha^{2}\right), \quad r \in(\eta, 1)
$$

satisfies the condition $-\left(\frac{1}{r^{2}}+\alpha^{2}\right)<0$, along with the simple separated boundary conditions associated with $\tilde{M}$. However, even more can be said ([14], [15]). Consider first order differential operators given by

$$
\begin{aligned}
\left(D_{j} \varphi\right)(r) & =\frac{d}{d r} w_{j}(r) \varphi(r), \\
\left(D_{j}^{*} \varphi\right)(r) & =w_{j}(r) \frac{d \varphi(r)}{d r}, j=1,2, \cdots, n,
\end{aligned}
$$

with strictly positive weights $w_{j}(r), j=1, \cdots, n+1$, possessing $2 n$ continuous derivatives in $[\eta, 1]$. The formal differential operators $A_{n}$ and $A_{n}^{*}$, which can be written as

$$
\begin{aligned}
& \left(A_{n} \varphi\right)(r)=D_{n} \cdots D_{2} D_{1} \varphi, \\
& \left(A_{n}^{*} \varphi\right)(r)=D_{1}^{*} D_{2}^{*} \cdots D_{n}^{*} \varphi,
\end{aligned}
$$

form factors of the even order differential operator

$$
E_{2 n}=\frac{(-1)^{n}}{r} A_{n}^{*} w_{n+1} A_{n}
$$

The appropriate boundary conditions for $E_{2 n}$ might be: at the endpoint $r=\eta$,

$$
\begin{gathered}
\left(D_{2}^{*} D_{3}^{*} \cdots D_{n}^{*} w_{n+1} D_{n} \cdots D_{2} D_{1} \varphi\right)(\eta)+(-1)^{n} a_{1} \varphi(\eta)=0, \\
\left(D_{3}^{*} D_{4}^{*} \cdots D_{n}^{*} w_{n+1} D_{n} \cdots D_{2} D_{1} \varphi\right)(\eta)+(-1)^{n+1} a_{2}\left(D_{1} \varphi\right)(\eta)=0,
\end{gathered}
$$

and at the endpoint $r=1$,

$$
\begin{gathered}
\left(D_{2}^{*} D_{3}^{*} \cdots D_{n}^{*} w_{n+1} D_{n} \cdots D_{2} D_{1} \varphi\right)(1)+(-1)^{n+1} b_{1} \varphi(1)=0, \\
\left(D_{3}^{*} D_{4}^{*} \cdots D_{n}^{*} w_{n+1} D_{n} \cdots D_{2} D_{1} \varphi\right)(1)+(-1)^{n+2} b_{2}\left(D_{1} \varphi\right)(1)=0, \\
\vdots \\
\left(w_{n+1} D_{n} \cdots D_{2} D_{1} \varphi\right)(1)+(-1)^{2 n} b_{n}\left(D_{n-1} \cdots D_{2} D_{1} \varphi\right)(1)=0
\end{gathered}
$$

where

$$
0 \leq a_{j} \leq \infty, \quad 0 \leq b_{j} \leq \infty, \quad j=1,2, \cdots, n
$$

and for each $j$ not both $a_{j}$ and $b_{j}$ are zero. (This restriction is to ensure that 0 is not an eigenvalue of $E_{2 n}$.) The value $a_{1}=\infty$ is interpreted as the boundary condition $\varphi=0$; similarly $a_{2}=\infty$ as the boundary condition $D_{1} \varphi=0$, etc. Then the Green's function kernel of $E_{2 n}, g_{2 n}$, is totally positive. This means that $g_{2 n}>0$ on the interior of the square on which it is defined, and certain determinants of intermediate values are 
nonnegative. It is only the positivity characterization which is needed for the rest of this discussion. As Yudovich [31] points out, $M^{*}$ has the factorization

$$
M^{*} \varphi=-\frac{1}{r I_{1}(\alpha r)} \frac{d}{d r}\left[r I_{1}^{2}(\alpha r) \frac{d}{d r} \frac{\varphi}{I_{1}(\alpha r)}\right]:=E_{2} \varphi .
$$

Thus it can be shown that the kernel function for $\tilde{I}^{-1}$ is totally positive. Likewise since $\left(M^{*} M\right) \varphi=E_{2} E_{2} \varphi:=E_{4} \varphi$, on $\operatorname{dom}\left(M^{*} M\right),\left(M^{*} M\right)^{-1}$ has a totally positive Green's function kernel. It also follows that $G\left(\sigma_{0}\right)=\left(M I^{*} M+\sigma_{0} M\right)^{-1}$ has a totally positive kernel for all real $\sigma_{0}>-\alpha^{2}$. since $-\left(M^{*}+\sigma_{0}\right)$ has the factorization

$$
-\left(M^{*}+\sigma_{0}\right) \varphi=\frac{1}{r I_{1}(\mu r)} \frac{d}{d r}\left[r I_{1}^{2}(\mu r) \frac{d}{d r} \frac{\varphi}{I_{1}(\mu r)}\right] .
$$

where $\mu=\sqrt{\sigma_{0}+\alpha^{2}}$. Together, (4.29) and (4.28) give that $G\left(\sigma_{0}\right)$ has a nonnegative kernel, when $\sigma_{0}$ is real, and $\sigma_{0}>-\alpha^{2}$. Hence, it is anticipated that the expansion (4.22) contains the desired result.

Consider then the term of order $-\sigma_{0}$ in (4.22). This is an integral operator defined on any function $\varphi \in H_{0}$. That is, set

$$
S=\tilde{M}^{-1}(I-Q) \tilde{M}^{-1}(I-Q) \tilde{M}^{-1} .
$$

It will be shown that $S$ has a nomnegative kernel. Let the kernel of $S$ be called $h$. We will write $h$ as the sum of two kernels satisfying conditions of the same form as (4.26) and (4.27). That is, $h=h_{1}+h_{2}$, where as functions of $r$

$$
h_{i}(\eta)=h_{i}^{\prime}(\eta)=h_{i}(1)=h_{i}^{\prime}(1)=0, \quad i=1,2 .
$$

Owing to the fact that $h$ ( and thereby $S$ ) represents the inverse of a sixth order operator $E_{6}$, two other boundary conditions must be imposed in order to determine it. From the nature of $S$, these conditions are

$$
\int_{\eta}^{1} r I_{1}(\alpha r)\left(\frac{d^{2}}{d r^{2}}+\frac{1}{r} \frac{d}{d r}-\left(\frac{1}{r^{2}}+\alpha^{2}\right)\right)^{2} h d r=0
$$

and

$$
\int_{\eta}^{1} r K_{1}(\alpha r)\left(\frac{d^{2}}{d r^{2}}+\frac{1}{r} \frac{d}{d r}-\left(\frac{1}{r^{2}}+\alpha^{2}\right)\right)^{2} h d r=0 .
$$

After the integrals are performed and the conditions (4.31) are imposed the other two conditions on $h$ become

$I_{1}(\alpha) h^{\prime \prime \prime}(1)+\left(2 I_{1}(\alpha)-\alpha I_{0}(\alpha)\right) h^{\prime \prime}(1)-\eta I_{1}(\alpha \eta) h^{\prime \prime \prime}(\eta)-\left(2 I_{1}(\alpha \eta)-\alpha \eta I_{0}(\alpha \eta)\right) h^{\prime \prime}(\eta)=0$,

$K_{1}(\alpha) h^{\prime \prime \prime}(1)+\left(2 K_{1}(\alpha)+\alpha K_{0}(\alpha)\right) h^{\prime \prime}(1)-\eta K_{1}(\alpha \eta) h^{\prime \prime \prime}(\eta)-\left(2 K_{1}(\alpha \eta)+\alpha \eta K_{0}(\alpha \eta)\right) h^{\prime \prime}(\eta)=0$, where $I_{0}$ and $K_{0}$ are the modified Bessel functions of order zero. However, these conditions are not of the same form as (4.26) and (4.27). In order to employ totally positivity theory, we search for such a decomposition, namely suppose that with $n=3, w_{1}=1 / I_{1}(\alpha r), w_{2}=r\left(I_{1}(\alpha r)\right)^{2}, w_{3}=1 / w_{2}, w_{4}^{\prime}=w_{2}$. Then suitable separated boundary conditions are

$$
\left(w_{4} D_{3} D_{2} D_{1} h_{i}\right)(\eta)-a_{i}\left(D_{2} D_{1} h_{i}\right)(\eta)=0
$$


and

$$
\left(w_{4} D_{3} D_{2} D_{1} h_{i}\right)(1)+b_{i}\left(D_{2} D_{1} h_{i}\right)(1)=0, \quad i=1,2 .
$$

Then with (4.31), these reduce to

$$
\begin{aligned}
\eta I_{1}(\alpha \eta) h_{i}^{\prime \prime \prime}(\eta)+\left(\left(2-a_{i} \eta\right) I_{1}(\alpha \eta)-\alpha \eta I_{0}(\alpha \eta)\right) h_{i}^{\prime \prime}(\eta) & =0, \\
I_{1}(\alpha) h_{i}^{\prime \prime \prime}(1)+\left(\left(2+b_{i}\right) I_{1}(\alpha)-\alpha I_{0}(\alpha)\right) h_{i}^{\prime \prime}(1) & =0, \quad i=1,2 .
\end{aligned}
$$

The choice of constants $a_{i} \geq 0$ and $b_{i} \geq 0$ must be made so that given $h=h_{1}+h_{2}$. (4.33) are compatible with (4.32). Such a decomposition will not be unique, but it can be done since (4.33) and (4.32) represent six homogeneous equations in the eight unknown boundary values. This system is compatible for nontrivial solutions as long as $a_{1} \neq a_{2}$ and $b_{1} \neq b_{2}$. Thus, it may be concluded that $S$ in (4.30) has a nomnegative kernel, so $S$ is a positive operator.

In a similar manner, the term of order $\sigma_{0}^{2}$ in (4.22) is representable as

$$
\tilde{M}^{-1}(I-Q) \tilde{M}^{-1}(I-Q) \tilde{M}^{-1}(I-Q) \tilde{M}^{-1} \text {. }
$$

This is obtainable from a differential operator $E_{8}$, that is with $n=4$ in (4.25). For the kernel of this operator perform a decomposition into two parts so that to each, (4.31) will apply. But now, not only do (4.32) apply as before, but a higher order counterpart as well. Through expressions such as (4.33), it is possible to find suitable separated boundary conditions at higher orders as well. The net decomposition is that the kernel for (4.34) is written as the sum of four nonnegative kernels. This renders (4.34) as a positive operator. By this procedure, each successive term in (4.22) is expressible as a sum of $2^{n-2}$ positive operators for $n=2,3,4, \ldots$ respectively. This means that for $\sigma_{0}$ real, and $\sigma_{0}>-\alpha^{2}$, each term is a positive operator.

What has been shown is that the expansion (4.16) for $G\left(\sigma_{0}\right)$ converges for $\left|\sigma_{0}\right|\|B\|_{0}<1$ and for $\sigma_{0}$ real, it gives that the operator $G\left(\sigma_{0}\right)$ is positive for $-\alpha^{2}<\sigma_{0}<\|B\|_{0}^{-1}$. Perform another expansion about a real point $\sigma_{1}>0$, such that $\sigma_{0}<\sigma_{1}<\|B\|_{0}^{-1}$. By summing the series for $G\left(\sigma_{1}\right)$ find that from (4.15),

$$
G\left(\sigma_{1}\right)=G\left(\sigma_{0}\right)\left[I+\left(\sigma_{0}-\sigma_{1}\right) M G\left(\sigma_{0}\right)+\left(\sigma_{0}-\sigma_{1}\right)^{2}\left(M G\left(\sigma_{0}\right)\right)^{2}+\ldots\right] .
$$

It has already been established that $G\left(\sigma_{0}\right)$ has positive coefficients in powers of $\left(-\sigma_{0}\right)$, when $-\alpha^{2}<\sigma_{0}<\|B\|_{0}^{-1}$, as in (4.16). By similar reasoning, it is observed that $G\left(\sigma_{1}\right)$ has the same properties as $G\left(\sigma_{0}\right)$ since, for example, the second coefficient $G\left(\sigma_{0}\right) \Lambda G\left(\sigma_{0}\right)$ in (4.35) behaves like a typical factor in (4.21) when expanded.

By Remark $3,-\|\Gamma(0)\|_{0}^{-1}=-\left\|\tilde{M}^{-1}\right\|_{0}^{-1}<-\alpha^{2}$, and by $(4.20)$, since $-\|B\|_{0}^{-1} \leq$ $-\left\|\tilde{M}^{-1}\right\|_{0}^{-1}<-\alpha^{2}$, the region of positivity of $G\left(\sigma_{1}\right)$ lies inside the region of analyticity given by (4.16). However, the only singularity on the edge of the disc of convergence for (4.16), as is shown in the expression for $G\left(\sigma_{1}\right)$ given by (4.14), would occur where $\sigma_{1}=-\|B\|_{0}^{-1}$, since $G(\sigma)$ has no singularities off the real line. Thus it can be concluded using analytic continuation that $G(\sigma)$ may be written as

$$
G(\sigma)=G\left(\sigma_{1}\right)\left[I+\left(\sigma_{1}-\sigma\right) M G\left(\sigma_{1}\right)+\left(\sigma_{1}-\sigma\right)^{2}\left(M G\left(\sigma_{1}\right)\right)^{2}+\ldots\right]
$$


where $\left|\sigma-\sigma_{1}\right|<\sigma_{1}+\|B\|_{0}^{-1}$. This disc goes outside the original disc $|\sigma|<\|B\|_{0}^{-1}$. The positivity is thus preserved for $\sigma_{0}$ real and $-\alpha^{2}<\sigma_{0}<2 \sigma_{1}+\|B\|_{0}^{-1}$. By a sequence of such discs, with singularities of (4.14) only at the (negative) eigenvalues of (4.12), any point in the half-plane $\operatorname{Re}(\sigma)>-\alpha^{2}$ can be covered. The positivity of the limiting operator thus holds for all real $\sigma_{0}>-\alpha^{2}$.

Computing the derivative expansions of $G(\sigma)$ in (4.15), we see that each term is of the form (4.22), so that $\left(-\frac{d}{d \sigma}\right)^{n} G\left(\sigma_{0}\right)$ is positive for all $n$ and $G(\sigma)$ has a power series expansion with positive coefficients.

5. Proof of PES. With the aid of Lemma 3, it is possible now to complete the abstract analysis of the earlier formulation (3.3) and obtain the desired result.

Theorem. The Principle of Exchange of Stabilities holds for (4.1)-(4.2), when the cylinders rotate in the same direction and the circulation decreases outwards.

Proof. The system (4.1)-(4.2) may be written as the single equation suggested by $(3.3)$.

$$
u=K(\sigma) u
$$

where

$$
K(\sigma)=\alpha^{2} T G(\sigma)\left[\left(\frac{1}{r^{2}}-\kappa\right) \Gamma(\sigma)\right] .
$$

The resolvent is examined as defined in (3.5). It has been demonstrated that the original system (4.1)-(4.2), and the transformed system (5.1), have spectra that agree except on the set $\Sigma_{\alpha}$. which is a subset of the negative real half-line. We have shown in Lemma 3 that $\Gamma(\sigma)$ and $G(\sigma)$ have power series expansions for real $\sigma_{0}>-\alpha^{2}$. To apply the Krein-Rutman Theorem (Sec. 3). we can take the Banach space to be the Hilbert space $H_{0}, \mathfrak{K}$, the cone of nomnegative functions in $H_{0}$, and the positive operator $A$ each of the operators $\left(-\frac{d}{d \sigma}\right)^{n} \Gamma\left(\sigma_{0}\right),\left(-\frac{d}{d \sigma}\right)^{n} G\left(\sigma_{0}\right), n=0,1, \ldots$, which are compact and linear.

To verify condition (P2). again note that $\left(\frac{1}{r^{2}}-k\right)$ does not change sign. while $\alpha^{2}$ and $T$ are positive. Therefore, by the product rule for differentiation, one concludes that $K(\sigma)$ in (4.36) satisfies condition (P2).

It has been demonstrated that all of the terms in $K^{-}(\sigma)$ determine positive operators. Moreover, for $\sigma$ real and sufficiently large, by Remark 3 and (4.14), the norms of the operators $\Gamma(\sigma)$ and $G(\sigma)$ become arbitrarily small. Hence, $\|K(\sigma)\|_{0}$ will be less than 1 . Then $[I-K(\sigma)]^{-1}$ has a convergent Neumam series and hence is positive. This is the content of condition (P1).

\section{REFERENCES}

[1] S. Chandrasekhar. Hydrodynamic and Hydromagnetic Stability. Oxford Clarendon Press, 1961.

[2] D. Coles, Transition in circular Couette flow, J. Fluid Mech. 21. 385-425 (1965).

[3] S. H. Davis, On the principle of exchange of stabilities, Proc. Roy. Soc. A. 310, $341-358$ (1969).

[4] R. C. DiPrima \& G. J. Habetler. A Completeness Theorem for Non-selfadjoint Eigenvalue Problems in Hydrodynamic Stability. Arch. Rat. Mech. Anal. 34. 218-227 (1969).

[5] R. C. DiPrima \& P. Hall. Complex eigenvalues for the stability of Couette flow, Proc. Roy. Soc. A. 396. 75-94 (1984). 
[6] G. F. D. Duff, Positive Elementary Solutions and Completely Monotonic Functions, Journ. Math. Anal. Appl. 27, 469-494 (1969).

[7] G. P. Galdi \& B. Straughan, Exchange of stabilities, symmetry and nonlinear stability, Arch. Rat. Mech. Anal. 89, 211-228 (1985).

[8] F. R. Gantmacher, Applications of the Theory of Matrices, Interscience, New York. 1959.

[9] I. H. Herron, Exchange of stabilities for Görtler flow, SIAM J. Appl. Math. 45, 775-79 (1985).

[10] H. Jeffreys, The stability of a layer of fluid heated from below, Phil. Mag. (7) 2, 833-44 (1926).

[11] D. D. Joseph, Stability of Fluid Motions, Vol I, Springer-Verlag, Berlin, 1976.

[12] D. D. Joseph \& W. Hung, Contributions to the nonlinear theory of stability of viscous flow in pipes and between rotating cylinders, Arch. Rat. Mech. Anal. 44, 1-22 (1971).

[13] S. Karlin, Positive operators, J. of Math. and Mech. 8, 907-37 (1959).

[14] S. Karlin, Total Positivity, Vol I, Stanford Univ. Press, Stanford, 1968.

[15] J. M. Karon, The Sign-Regularity Properties of a Class of Green's Functions for Ordinary Differential Equations, J. Diff. Eqns. 6, 484-502 (1969).

[16] T. Kato, Perturbation Theory for Linear Operators, 2nd ed., Springer-Verlag, Berlin (1976).

[17] K. Kirchgässner, Bifurcation in Nonlinear Hydrodynamic Stability, SIAM Rev. 17, 652-683 (1975).

[18] M. G. Krein \& M. A. Rutman, Linear operators leaving invariant a cone in a Banach space, Uspekhi Matem. Nauk 3, 3-95 (1948) (A.M.S. Transl. No. 26 (1950))

[19] J. W. Lewis, An experimental study of the motion of a viscous liquid and contained between two coaxial cylinders, Proc. Roy. Soc. London A 117, 388-407 (1928).

[20] W. S. Loud, Some generalized Green's functions and generalized Green's matrices, SIAM Rev. 12, 194-210 (1970).

[21] P. H. Rabinowitz, Nonuniqueness of Rectangular Solutions of the Bénard Problem, in Bifurcation Theory and Nonlinear Eigenvalue Problems, Keller, J. B. \& Antman, S. eds., Benjamin, New York, 1969.

[22] G. Schneider, Nonlinear Stability of Taylor Vortices in Infinite Cylinders, Arch. Rat. Mech. Anal. 144, 121-200 (1998).

[23] J. L. Synge, On the stability of a viscous liquid between rotating coaxial cylinders, Proc. Roy. Soc. A. 167, 250-56 (1938).

[24] R. Tagg, The Couette-Taylor problem, Nonlinear Sci. Today 4, No. 3, 1-25 (1994).

[25] G. I. Taylor, Experiments with rotating fluids, Proc. Camb. Phil. Soc. 20, 326-9 (1921).

[26] G. I. Taylor, Stability of a viscous fluid contained between two rotating cylinders, Phil. Trans. A. 233, 289-343 (1923).

[27] W. Velte, Stabilatät und Verzweigung stationärer Lösungen der Navier-Stokesschen Gleichungen beim Taylor-Problem, Arch. Rat. Mech. Anal. 22, 1-14 (1966).

[28] H. F. Weinberger, Exchange of stability in Couette flow, in Bifurcation Theory and Nonlinear Eigenvalue Problems, Keller, J. B. \& Antman, S. eds., New York: Benjamin, 1969.

[29] C.-S. Yih, Spectral Theory of Taylor vortices, I and II, Arch. Rat. Mech. Anal. 46, 218-240, and 47, 288-300 (1972).

[30] V. I. Yudovich, The bifurcation of a rotating flow of a liquid, Sov. Phys. Dok. 11, 566-568 (1966/67).

[31] V. I. Yudovich, Secondary flows and fluid instability between rotating cylinders, J. Appl. Math. Mech. 30, 822-833 (1966). 